\title{
ANDES

www.scielo.cl

\section{Megacolon Tóxico: complicación infrecuente de la constipación crónica. A propósito de 3 casos pediátricos}

\author{
Toxic Megacolon: A rare complication of chronic constipation. \\ Three Pediatric Cases
}

\author{
Macarena Muñoz P.a,b, Fernanda Andueza A. ${ }^{\mathrm{a}, \mathrm{b}}$, Marcela Santos M. ${ }^{\mathrm{a}}$
}

aServicio de Cirugía Pediátrica, Hospital Exequiel González Cortés, Universidad de Chile. Santiago, Chile

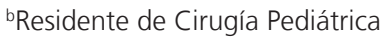

Recibido: 16 de noviembre de 2020; Aceptado: 24 de junio de 2021

¿Qué se sabe del tema que trata este estudio?

El Megacolon tóxico es una patología infrecuente, pero de alta morbimortalidad, que puede resultar como complicación de patologías infecciosas e inflamatorias que afectan el colon. Una causa infrecuente es la constipación crónica con mala adherencia, o sin tratamiento.

\section{¿Qué aporta este estudio a lo ya conocido?}

Visibilizar al Megacolon tóxico como una complicación de la constipación crónica en la edad pediátrica, y la necesidad de su manejo multidisciplinario precoz, donde el equipo quirúrgico es parte del tratamiento y no sólo una medida de rescate.

\section{Resumen}

La constipación crónica es una patología frecuente en la edad pediátrica, que afecta la calidad de vida de los pacientes y su familia. Su manejo suele ser complejo y prolongado. El megacolon tóxico es una patología grave, potencialmente letal, que puede derivar de una constipación crónica no tratada o con mala adherencia. Objetivo: Comunicar 3 casos pediátricos de megacolon tóxico como complicación de una constipación crónica de mal manejo. Casos Clínicos: Se presentan 3 pacientes, edad 6 a 13 años, de sexo masculino, con historia de constipación crónica con mala adherencia a tratamiento. Ingresaron a Servicio de urgencia por cuadro característico de megacolon tóxico (dilatación intestinal y signos de toxicidad sistémica). Dada su inestabilidad, requirieron manejo en unidad de paciente crítico e intervención quirúrgica precoz, siendo ostomizados. Todos presentaron evolución favorable, siendo reconstituidos entre 8-24 meses después. En todos los casos se descartó patología orgánica como causa de su constipación. Conclusión: El megacolon tóxico es una patología poco frecuente pero de alta morbilidad y potencialmente letal. Requiere de un alto índice de sospecha así como un manejo multidisciplinario médico quirúrgico.
Palabras clave: Megacolon Tóxico; Constipación; Procedimientos Quirúrgicos; Ostomia; Colostomia 


\begin{abstract}
Chronic constipation is a frequent pathology in the pediatric age that affects the quality of life of patients and their families. Its management is usually complex and long associated with poor adherence. Toxic megacolon is a serious, potentially lethal disease when chronic constipation is left untreated or poor adherence to treatment. Objective: To report 3 pediatric cases of toxic megacolon as a complication of poorly managed chronic constipation. Clinical Cases: Three males patients, aged 6 to 13 years, with a history of chronic constipation and poor adherence to treatment are discussed. They were admitted to the emergency department with clinical findings of toxic megacolon (intestinal dilation and signs of systemic toxicity). Given their condition, all patients required management in the critical patient unit (CPU) and early surgical intervention, undergoing ostomy. All presented favorable outcome, performing stoma reversal surgery between 8-24 months later. In all cases, organic cause of the constipation was ruled out. Conclusions: Toxic megacolon is an infrequent but highly morbid and potentially lethal disease. It requires a high index of suspicion as well as multidisciplinary medical-surgical management.
\end{abstract}

\section{Keywords:}

Toxic Megacolon;

Constipation;

Surgical Procedures;

Ostomy;

Colostomy

\section{Introducción}

La constipación crónica es un problema frecuente en la edad pediátrica, con una prevalencia de 0,7 $29,6 \%{ }^{1,2}$, describiéndose como un $25 \%$ las consultas en gastroenterología $a^{3,4}$. Su fisiopatología es multifactorial, en un $90-95 \%$ de los casos de causa funcional ${ }^{2-5}$. Es necesario una acuciosa anamnesis y examen físico en búsqueda de antecedentes que pudieran orientar a patología orgánica, incluyendo momento de eliminación de meconio, coexistencia de enuresis e infecciones urinarias, así como el consumo de medicamentos ${ }^{2,3}$.

Se ha observado que los niveles socioeconómicos bajos tendrían relación con mayores prevalencias de constipación crónica, así como también una relación inversa con el número de años de estudios de los padres $^{1}$. Otros factores de riesgo serían el sedentarismo, el bajo consumo de fibra, frutas y verduras, vivir en zonas de mayor densidad poblacional, historia familiar de constipación, eventos de vida estresantes y depre$\operatorname{sión}^{1,3,5}$.

Esta patología no sólo tiene implicancias económicas, debido al costo asociado a las consultas médicas, sino que también por atenciones en unidad de emergencia y gasto en medicamentos ${ }^{1}$. La constipación crónica impacta la calidad de vida de manera comparable a otras enfermedades crónicas, afectando el desempeño escolar y generando ausentismo ${ }^{6}$. Su manejo está orientado a educación, cambios en la dieta, formación de hábito defecatorio, medidas de desimpactación fecal y mantenimiento de hábito defecatorio ${ }^{3}$. Un 25$30 \%$ de los pacientes continúan sintomáticos hasta la vida adulta ${ }^{2,3}$.

El Megacolon Tóxico (MT) es una patología grave, de múltiples causas, una de ellas, la constipación crónica no tratada o con pobre adherencia a tratamiento, de la cual existe poca literatura en pediatría. Pese a que no se encontraron reportes específicos de MT asociados a constipación crónica, se describe una mortalidad variable asociada a esta patología, que puede llegar hasta $45 \%$, según la serie analizada ${ }^{6,11}$.

El objetivo de este reporte es comunicar 3 casos pediátricos de megacolon tóxico como complicación de una constipación crónica de mal manejo.

\section{Casos Clínicos}

\section{Caso 1}

Niño de 13 años con antecedente de constipación crónica desde los 3 años (deposiciones 3 veces por semana y escurrimiento diario), con mala adherencia a tratamiento. Consultó por cuadro de fiebre, dolor abdominal, compromiso del estado general, grave, con cuadro compatible con shock séptico de posible foco abdominal de difícil manejo hemodinámico, por lo que fue ingresado a unidad de paciente crítico (UPC). Ecografía de abdomen evidenció distensión patológica de colon de hasta $5,5 \mathrm{cms}$, con abundante contenido interior y distensión patológica de ampolla rectal. Laboratorio: Hemocultivo (+) E. coli multisensible, coprocultivos y urocultivos negativos. Se realizó laparatomía exploradora, apreciándose gran compromiso isquémico necrótico colónico, por lo que, dada la gravedad del paciente, se realizó ileostomía (en asa a $20 \mathrm{cms}$ de válvula ileocecal) y colectomía subtotal hasta sigmoides con técnica de Hartmann. Tras 3 cirugías egresó en buenas condiciones, realizando reconstitución intestinal 2 años después.

\section{Caso 2}

Niño de 12 años con antecedente de constipación crónica desde los 6 años (deposiciones diarias, escurrimiento diario), con mala adherencia a tratamiento. Hipotiroidismo en tratamiento. Ingresó al Servicio de 
Urgencia (SU) por cuadro de $24 \mathrm{hrs}$ de diarrea profusa y vómitos, compromiso de conciencia cualitativo, en malas condiciones, mal perfundido y abdomen distendido. Evolucionó con shock mixto hipovolémico-séptico y disfunción multiorgánica, requiriendo manejo en UPC. Tomografía axial computada (TAC) de abdomen evidencia asas colónicas dilatadas, con pérdida de la haustración normal (figuras 1-3). Laboratorio: Hemocultivo, Coprocultivo y Urocultivo negativos, estudio Chagas negativo. Se realizó laparoscopía exploradora e ileostomía en asa, videoasistida, egresando 2 semanas después. Reconstitución intestinal a los 2 años.

\section{Caso 3}

Niño de 6 años con antecedente de constipación desde el año de vida (no se describe historia de deposiciones). Sin adherencia a tratamiento. Consultó por cuadro de deposiciones liquidas, deterioro progresivo del estado general y posterior compromiso de conciencia. Ingresó a SU en malas condiciones generales, gran distensión abdominal, y sospecha de shock séptico de foco abdominal que requirió manejo en UPC. Radiografía de abdomen muestra importante distensión de colon (figuras 4 y 5). Laboratorio: Hemocultivo, Coprocultivo y Urocultivo negativos. Laparotomía exploradora evidenció ileon distal y colon distendidos, sin compromiso isquémico. Se realizó ileostomía en asa aproximadamente a $15 \mathrm{~cm}$ de válvula ileocecal. Reconstitución intestinal a los 8 meses.

En los 3 casos se realizó estudio anatomo-patológico que evidenció histoarquitectura conservada, reconociéndose presencia de plexo submucoso y mientérico normogangliónico, sin hiperplasia de troncos nerviosos, descartándose Enfermedad de Hirschsprung y Enfermedad Inflamatoria Intestinal.
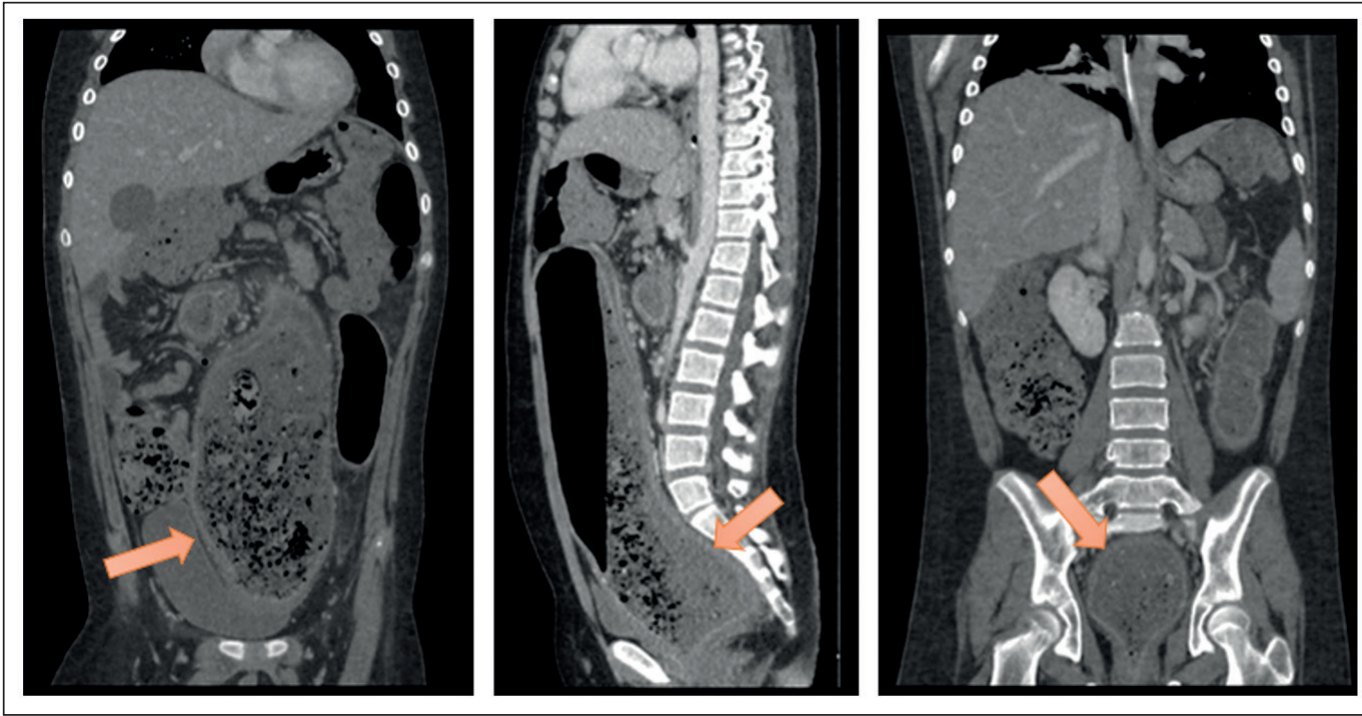

Figuras 1,2 y 3 . Caso clínico 2. TAC de abdomen y pelvis, importante dilatación de colon izquierdo y recto sigmoides con contenido fecal en su interior y pérdida de haustración normal.

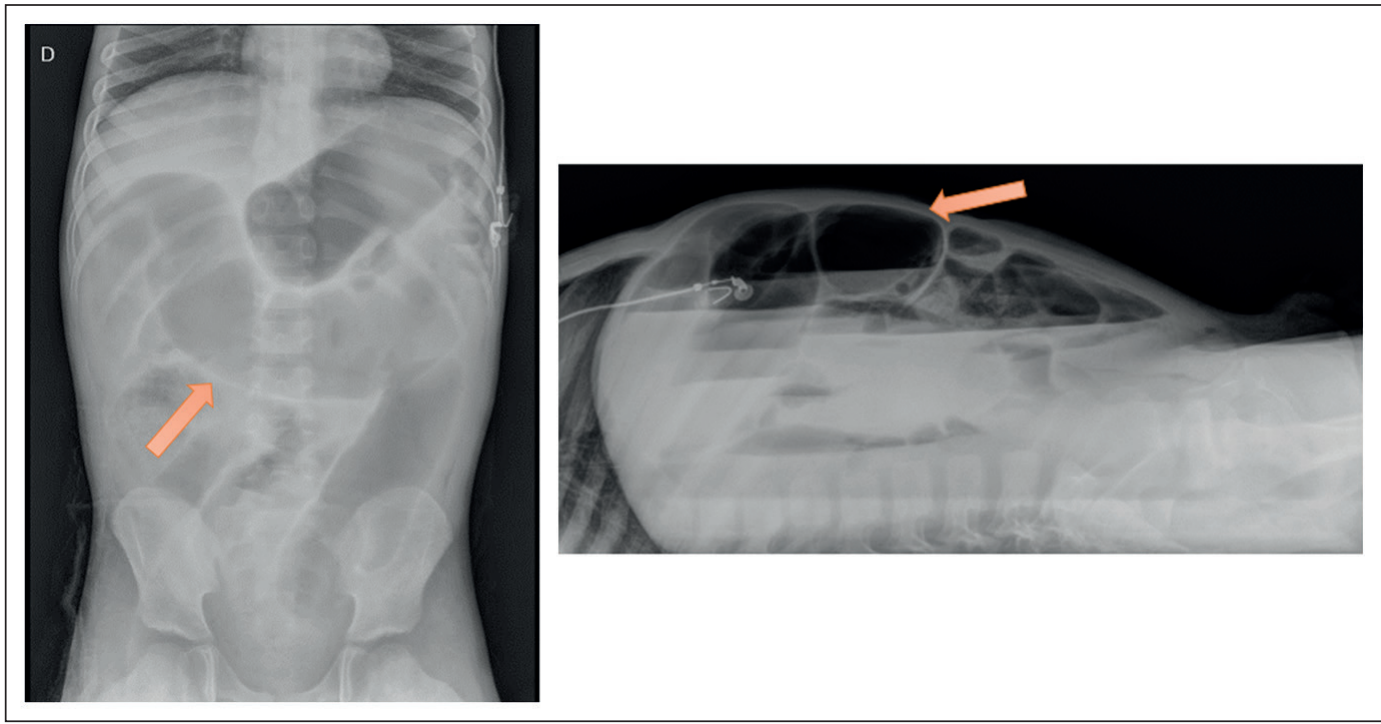

Figura 4 y 5. Caso Clínico 3. Radiografías de ingreso, se aprecia dilatación de Colon y pérdida de las haustración normal. 


\section{Discusión}

El tratamiento de la constipación crónica es complejo, incluyendo educación al paciente y sus cuidadores, cambios en hábitos alimenticios y medidas farmacológicas ${ }^{3}$. Es primordial lograr el compromiso del paciente y su familia, ya que el tratamiento es prolongado, y pueden presentarse fracasos a las distintas estrategias utilizadas, perdiéndose la adherencia al mis$\mathrm{mo}^{3}$.

En nuestro medio, la mayoría de los pacientes son atendidos en el nivel primario de atención, siendo derivados al nivel secundario y terciario de salud (Pediatría y Cirugía Infantil) cuando existe sospecha de patología orgánica o mala respuesta al tratamiento disponible.

Pese a la prevalencia de esta patología, vemos con frecuencia peregrinación de los pacientes por distintos profesionales en búsqueda de una solución, consultando en la red pública y privada, así como en las unidades de emergencia por episodios de constipación "agudos sobre su condición de base", que además de aumentar los costos en salud, implican un evento traumático en los pacientes pediátricos que deben ser sometidos a un examen físico que implica tacto rectal y muchas veces enemas evacuantes.

El MT se define por dilatación colónica asociado a signos clínicos de toxicidad sistémica ${ }^{8-11}$. Se asocia de manera más frecuente a enfermedad inflamatoria intestinal, colitis infecciosa, enfermedad de Hirschsprung $^{9-10}$, pero también puede ser secundario al estadio final de una constipación crónica refractaria, con mala adherencia a tratamiento o ausencia de manejo ${ }^{12}$. Cabe destacar que, durante la revisión bibliográfica realizada para este manuscrito, no se encontraron reportes de casos de MT secundario a constipación en pacientes pediátricos.

Debido a que esta complicación potencialmente letal es infrecuente, puede ser subdiagnosticada ${ }^{9}$, requiriendo de un alto índice de sospecha por parte de los equipos de salud. El cuadro puede presentarse con dolor abdominal, diarrea y vómitos. Al examen físico destaca un abdomen sensible y distendido, ruidos intestinales disminuidos, fiebre, taquicardia, hipotensión, compromiso de conciencia9.

El MT requiere un reconocimiento precoz, así como un manejo multidisciplinario intensivo que incluya al equipo de Cirugía Infantil como parte integral y no tan sólo para el manejo de las complicaciones, tal como se demuestra en la serie de casos presentados. Existe controversia sobre el momento en que está indicada la cirugía en el MT. El tratamiento médico busca disminuir la necesidad intervención quirúrgica, sin embargo, retrasarla puede implicar complicaciones como perforación intestinal o síndrome compartimental abdominal ${ }^{10,11}$, reportándose aumento de la mortalidad en los casos en que se retrasa la cirugía ${ }^{11}$.

En conclusión, el MT es una patología grave, de múltiples causas, una de ellas la constipación crónica. Se asocia a alta morbimortalidad. El éxito de su tratamiento depende de la detección precoz, así como el manejo multidisciplinario oportuno que incluya al equipo quirúrgico. El tratamiento quirúrgico debe considerarse parte del tratamiento y no sólo como una medida de rescate, detectando así aquellos pacientes que se beneficiarán de la cirugía para disminuir la morbimortalidad, reintervenciones quirúrgicas y el número de días de hospitalización.

\section{Responsabilidades Éticas}

Protección de personas y animales: Los autores declaran que los procedimientos seguidos se conformaron a las normas éticas del comité de experimentación humana responsable y de acuerdo con la Asociación Médica Mundial y la Declaración de Helsinki.

Confidencialidad de los datos: Los autores declaran que han seguido los protocolos de su centro de trabajo sobre la publicación de datos de pacientes.

Derecho a la privacidad y consentimiento informado: Los autores han obtenido el consentimiento informado de los pacientes y/o sujetos referidos en el artículo. Este documento obra en poder del autor de correspondencia.

\section{Conflicto de intereses}

Los autores declaran no tener conflicto de intereses.

\section{Referencias}

1. Mugie S, Benninga M, Di Lorenzo C. Epidemiology of constipation in children and adults: A systematic review. Best Practice \& Research Clinical Gastroenterology 2011;25(1):318.
2. Agarwal J. Chronic Constipation. Indian J Pediatr. 2013;80(12):1021-5.

3. Torres A, González M. Constipación crónica. Rev Chil Pediatr. 2015;86(4):299304.

4. Khan A, Vujanic G, Huddart S. The constipated child: how likely is Hirschsprung's disease? Pediatr Surg Int.
2003;19:439-42.

5. Rubin G, Dale A. Chronic constipation in children. Clinical Review. BMJ 2006;333:1051-5.

6. Present D. Toxic Megacolon. Med Clin North Am. 1993;77(5):1129-48.

7. Belsey J, Greenfield S, Candy D, et al. Systematic review: impact of constipation 
on quality of life in adults and children. Aliment Pharmacol Ther. 2010;31:938-49.

8. Chiu C, Su L, He C, et al. Perforation of toxic megacolon in non-typhoid Salmonella enterocolitis spares young infants and is immune-mediated. Pediatric Surg Int. 2002;18:410-2.
9. Suita S, Sakaguchi T, Arima T, et al. Toxic megacolon in Hirschsprung's disease is still potentially fatal. Pediatr Surg Int. 1994;9:433-35.

10. Autenrieth D, Baumgart D. Toxic Megacolon. Clinical Review. Inflamm Bowel Dis. 2012;18(3):584-91.
11. Ausch C, Madoff D, Gnant M, et al. Aetiology and surgical management of toxic megacolon. Colorectal Dis. 2006;8(3):195-201.

12. Bharucha A, Phillips S. Megacolon: Acute, Toxic, and Chronic. Curr Treat Options Gastroenterol. 1999;2(6):517-23. 